\title{
Porous silicene and silicon graphenylene-like surfaces: a DFT study
}

\author{
G. S. L. Fabris ${ }^{1}$ N N. L. Marana ${ }^{1}$ E. Longo ${ }^{2}$ J. R. Sambrano ${ }^{1}$ \\ Received: 30 October 2017 / Accepted: 19 December 2017 / Published online: 5 January 2018 \\ c) Springer-Verlag GmbH Germany, part of Springer Nature 2018
}

\begin{abstract}
Nanoporous single-layers surfaces derived from silicene, named porous silicene (PS) and silicenylene (SC) have been studied via periodic density functional theory with a modified B3LYP functional combined with an all-electron Gaussian basis set. The structural, elastic, electronic and vibrational properties of these nanoporous surfaces were simulated and analyzed. The results show that both PS and SC structures had a non-null band gap and a buckled structure such as pristine silicene, besides that they are more susceptible to longitudinal and transversal deformation than silicene. The large and well-defined porous diameter of PS and SC can bring new applications, such as gas separation, filtering and as anode material for lithiumion batteries. These results are a challenge for the experimentalists to synthetize these new nanomaterials, comparing their properties with those described in this work.
\end{abstract}

Keywords Nanoporous · Silicene $\cdot$ Graphene-like $\cdot$ Porous silicene $\cdot$ Modified functional · B3LYP*

\section{Introduction}

This paper belongs to a collaborative project with Professor Claudio Zicovich-Wilson, initialized during one of his visit to Brazil. During his stay, our particular interest was devoted to the computational modeling of organic and inorganic nanoporous surfaces as described below.

The improvement and the level of chemical synthesis have reached a high sophistication and can bring new and interesting discovery in the nanotechnology field.

Materials with similar structures of graphene, called graphene-like (honeycomb network), have been studied and applied in several areas of science, but specially in the case of zero band gap materials their applications in

Published as part of the special collection of articles "In Memoriam of Claudio Zicovich."

Electronic supplementary material The online version of this article (https://doi.org/10.1007/s00214-017-2188-6) contains supplementary material, which is available to authorized users.

J. R. Sambrano

sambrano@fc.unesp.br

1 Modeling and Molecular Simulation Group - CDMF, São Paulo State University, P.O. Box 17033-360, Bauru, SP, Brazil

2 Chemistry Institute - CDMF, Federal University of São Carlos, P.O. Box 14801-907, São Carlos, SP, Brazil semiconductor devices are still problematic. Consequently, there is an increment in the search of chemical [1-4] and physical [5-7] methods with the aim of modifying the band gap on this class of materials.

For this reason, plausible alternatives, such as inorganic graphene-like structures, can bring new possibilities. In IV-group elements, such as silicon and germanium, there is some chemical similarity to carbon; therefore, they can form graphene-like structures, named as silicene $[8,9]$ and germanene [10], both with zero band gap.

The main difference between the last two structures and the graphene is that $\mathrm{Si}$ and $\mathrm{Ge}$ have larger ionic radius, which promotes $\mathrm{sp}^{3}$ hybridization, differently from $\mathrm{C}$ since $\mathrm{sp}^{2}$ hybridization is more energetically favorable; this enables the silicene bonding to be formed by a mixed $\mathrm{sp}^{2}$ and $\mathrm{sp}^{3}$ hybridization. The silicene has one of the two sublattices being displaced vertically with respect to the other, which is called buckling. The planar version of the silicene (where all atoms lie on the same level) is metastable [11] and it is not found in this form of deposition.

In the graphene-like structures each hexagon can be considered as nanoporous with well-defined characteristics. Classically, porous materials are organic materials with cavities, channels or interstices such as zeolites [12], metal organic frameworks (MOFs) [13], silicates [14] and based carbon materials [15]. The pore morphology of these materials can bring different characteristics, with special attention 
to the ratio of the total volume (area) of the pore and the apparent volume (area) of material.

In case of nanoporous structures derived from graphene and with well-defined porous network, the porous graphene (PG) [16-20] and graphenylene (GP) can be cited [20-23]. Unlike the graphene, both PG and GP have a non-null band gap, $\sim 3$ and $\sim 0.8 \mathrm{eV}$, respectively, as shown in recent work [20]. The non-null band gap could bring new possibilities of its applications in electronics.

A few years ago, Yu [24] showed that GP has a great potential as anode material for lithium-ion batteries and exhibits high storage capabilities; in addition, Song et al. [25] reported that GP can be used as molecular sieve for gas separation, since it has larger pore diameter than graphene, 5.47 and $2.83 \AA$, respectively.

Nowadays, silicon porous materials have also attracted attention from researchers, especially the silicene-based ones. The designed nanopores in silicene membranes with Stone Walles defects were presented by $\mathrm{Hu}$ et al. [26, 27]. These authors show that such nanopores have a high capability as helium gas separator and as hydrogen purification membrane, suggesting that these nanoporous materials might have great potential in gas separation and filtering applications.

Recently, Zhuang et al. [28] showed that the recent progress on the features of silicene makes it a promising anode for lithium-ion batteries. In another work, Zhang et al. [29] showed that a 3D interconnected porous structure of silicene, called silicene flowers, exhibits extraordinary combined performance for lithium battery anodes, including high gravimetric capability, high volumetric capacity, remarkable rate capability, and excellent cycling stability.

In order to better understand, the electronic characteristics of new nanoporous materials whose results can give an indication for possible applications, two nanoporous single-layer structures derived from silicene, named as porous silicene (PS) and silicenylene (SC) are here presented for the first time. These proposed structures are computationally studied under density functional theory framework, and an accurate investigation on their structural, elastic, electronic, and vibrational properties is reported.

\section{Computational methods}

The simulations were performed under periodic Density Functional Theory (DFT) in the CRYSTAL14 [30] program. In order to reach a reasonable accuracy on structural and electronic results, eight different functional have been considered and their results suitably compared: BLYP, B3LYP, B3LYP* (12\% hybrid), B3LYP** (6\% hybrid), PBE, PBE0, PBESOL0 and HSE06. The hybrid percentage means that the mixing of the exact
Hartree-Fock and DFT exchange are modified. The equation that describes the modified B3LYP functional is: $\quad E_{x c}=(1-A) \cdot\left(E_{x}^{L D A}+0.9 E_{x}^{B E C K E}\right)+A \cdot E_{x}^{H F}+0.19$. $E_{c}^{L D A}+0.81 \cdot E_{c}^{G G A}$, where $A$ is the hybrid percentage $(A=0.12$ or $A=0.06$ for B3LYP* and B3LYP**, respectively).

The atom centers were described by; 86-311G** [31] and 5-11G* [32] all-electron basis sets for silicon and hydrogen atoms, respectively.

The accuracy of the convergence criteria for bi-electronic integrals was controlled by a set of five thresholds $\left(10^{-10}\right.$, $\left.10^{-10}, 10^{-10}, 10^{-20}, 10^{-40}\right)$. These parameters represent the overlap and penetration for Coulomb integrals, the overlap for HF exchange integrals, and the pseudo-overlap, respectively, and the shrinking factor (Pack-Monkhorst and Gilat net) were set to 20 .

All stationary points were characterized as minimum diagonalizing the Hessian matrix regarding atomic coordinates and unit cell parameters and analyzing the vibrational modes at the $\Gamma$ point using the numerical second derivatives of the total energies estimated with the coupled perturbed Hartree-Fock/Kohn-Sham algorithm [33-35]. The convergence was checked on gradient components and nuclear displacements with tolerances on their root-mean-square set to 0.0001 and 0.0004 a.u., respectively.

The band structure and density of states (DOS) were analyzed using the Properties 14 routine implemented in the CRYSTAL code, using the same $\mathrm{k}$ point sampling employed for the diagonalization of the Fock matrix in the optimization process.

The elastic properties were also studied, and the elements of the elastic constant tensor $\left(C_{v u}\right)$ were calculated using the following equation with Voigt's notation:

$C_{v u}=\left.\frac{1}{V} \frac{\partial^{2} E}{\partial \eta_{v} \partial \eta_{u}}\right|_{0}$

where $V$ is the equilibrium unit cell volume, $\eta$ is the symmetric second-rank pure strain tensor. Second derivatives were computed as numerical derivatives of analytical energy gradients. In a surface (2D systems), due to the symmetry, there are only two elastic constants, labeled $C_{11}$ and $C_{12}$, which represent the longitudinal compression and transverse expansion, respectively.

It is important to note that in the case of $2 \mathrm{D}$ systems, the equation above needs to be modified and adapted to when the area replaces the volume. For this reason, the conventional units of 2D elastic constants $\left(\mathrm{Ha} \mathrm{bohr}^{2}\right)$ are different than units of the 3D elastic constants $(\mathrm{Pa})$.

Due to the 2D structure of the present systems and in order to enable the comparison of the used units, the constants were converted to gigapascal. Thus, the volume can be approximated using van der Waals radii and the unit cell area. 


\section{Model systems}

The silicon bulk has a cubic structure described by the Fd-3 m symmetry group with a diamond form and unit cell parameter $\boldsymbol{a}=5.431 \AA$ [36] with a band gap of $1.17 \mathrm{eV}$ [37]. Silicene is formed by a single flat layer of silicon atoms linked in a hexagonal shape described by the P-3m1 symmetry group and unit cell parameter $\boldsymbol{a}=3.830 \AA$ [38] with zero band gap and differently from graphene, presents a small buckling $\Delta=0.44 \AA$ [38].

In order to construct the nanoporous silicene singlelayer surface, the optimization of the lattice parameters and internal coordinates was firstly done to minimize the total energy of both diamond silicon and silicene single-layer. It is important to notice that the silicene is not obtained directly from the bulk structure, but rather obtained by deposition on metallic substrate, such as $\mathrm{Ag}[39,40]$ and $\mathrm{ZrB}_{2}$ [41].

The porous silicene (PS) is achieved by the replacement of each atom of the silicene unit cell by a benzene-like ring with three atoms of hydrogen alternated between two non-consecutive silicon atoms. In another way, the silicenylene (SC) is obtained by the replacement of each atom of the silicene unit cell by a dimer of benzene-like ring of silicon atoms.

Also, the SC could be a spontaneous interconversion of the PS structure without the hydrogen atoms, leading the rings to rotate with a simultaneous contraction of its lattice parameter, forming a cyclobutadiene-like ring between the dimer of benzene-like ring of silicon atoms [21]. Figure 1 shows the structures of silicene, PS and SC.

\section{Results and discussion}

\subsection{Structural properties}

The full optimization of structural parameters of the diamond silicon and silicene single-layer was done using the eight different functional mentioned above, whose results with the respective non-null band gap and buckling are described in Table 1.

As it is possible to see in Table 1, the description of the $\boldsymbol{a}$ silicon lattice parameter was done with relative accuracy for all selected functional and almost without errors for PBE0 and HSE06.

However, the band gap energy is poorly described (overestimated) for most of functional, except for B3LYP** and HSE06 that do the best description. For the silicene, all the functional gives a good description of the $\boldsymbol{a}$ lattice parameter, although only the buckling B3LYP* and B3LYP** gives an accurate result.
The motivation to modify the B3LYP functional urges from the need to have a better balance between the description of the structural parameters and the band gap. This modification can often improve the results giving a higher reliability, since an accurate description of these parameters is important to simulate and determine the possible applications of the material.

The need to accurately describe the lattice parameters and the band gap can be achieved by different methodologies, such as functional tests, basis set, optimization of basis set and/or functional change (as in the hybrid functional). All these tests bring with them a great computational cost, especially in the case of the simultaneous optimization of the basis set for each functional tested. For this reason, the methodology of fixing the basis set and the hybrid functional was successfully adopted, searching the best parameter adjustment for the mixing of the exact Hartree-Fock and DFT exchange. The last methodology was also successfully applied to study the phonons of $\mathrm{NiWO}_{4}$ [43] and other porous materials [20].

Moreover, it should be highlighted that the HSE06 functional produces a good approximation to structural parameters and also to the band gap. This functional takes into account the combination of short-range fragment of Fock exchange with a semilocal model of long-range exchange and can be considered as a reparametrization of the HSE functional $[44,45]$. Besides, this functional would be important if applied to van der Waals surfaces, where the interaction between the layers are important, although this is not the aim of the present study, since only single-layer systems were considered. Moreover, the buckling is not accurately described for this functional.

Based in the above discussion, the B $3 \mathrm{LYP} * *$ functional shows the best description for the lattice parameters and band gap energy of both diamond silicon and silicene singlelayer surface.

In order to maintain the simulation consistency, it was decided that the computational setup previously selected should be maintained. As the selected functional B3LYP** produces a good description of the support structures, it can also predict the properties of PS and SC with good accuracy, giving us plausible results with consistency and reliability.

The full structural optimization procedure shows that the PS and SC keep the hexagonal structure, with lattice parameters $\boldsymbol{a}=\boldsymbol{b}$ (see Table 2). The calculated lattice parameters of PS are $11.71 \AA$, whereas the parameters of the SC structure are $10.59 \AA$. Consequently, the SC structure presents a 9.5\% lattice contraction if compared with PS. The cell parameters and buckling, bond lengths and bond angles for PS and SC structures are summarized in Table 2.

The space group and unit cell coordinates were determined for both PS and SC nanoporous structures. PS belongs to the P6mm symmetry group with three irreducible atoms 
Fig. 1 Side and top view of nanoporous single-layer unit cell of a Silicene, $\mathbf{b}$ PS and c SC, with atom labels also indicated (a)
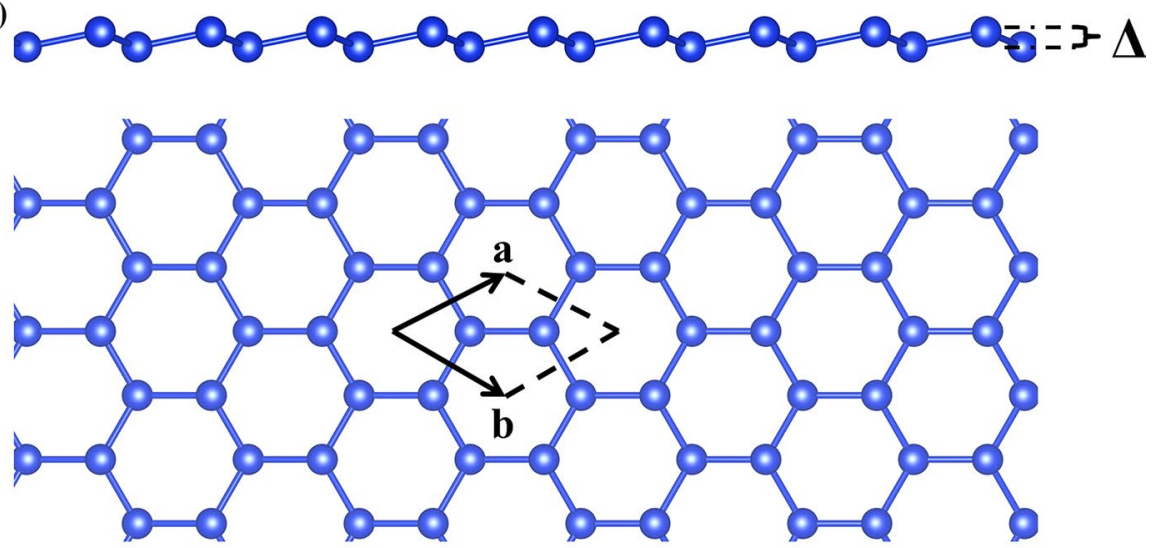

(b)
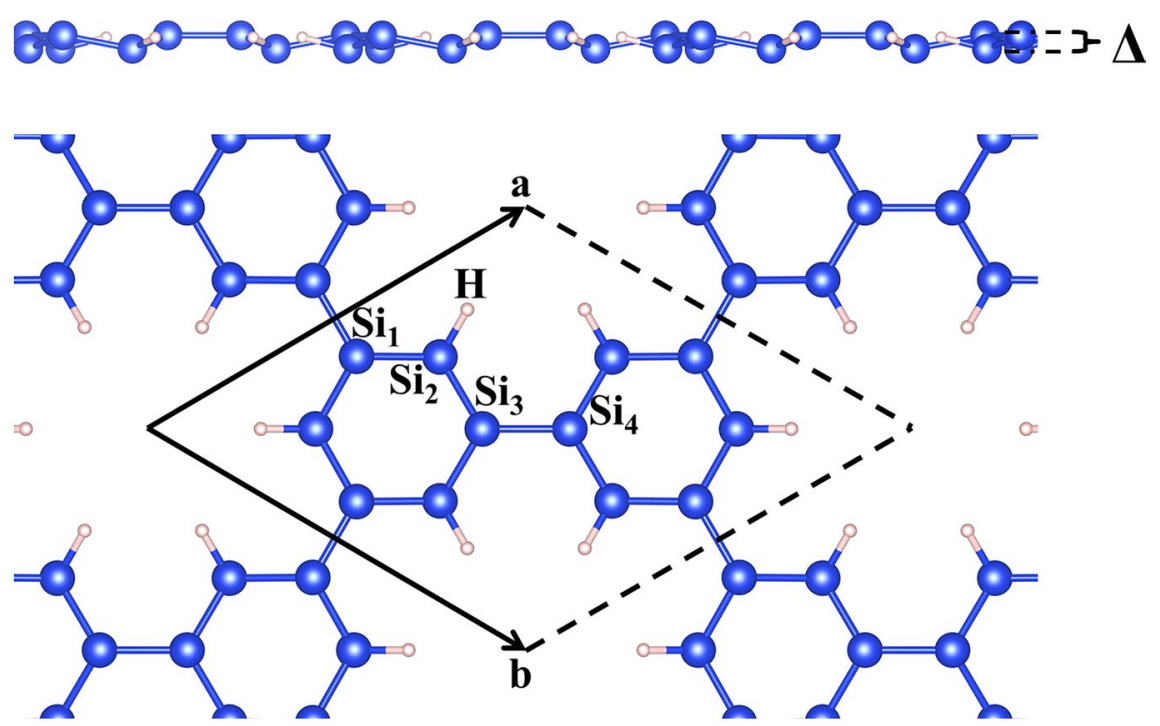

(c)
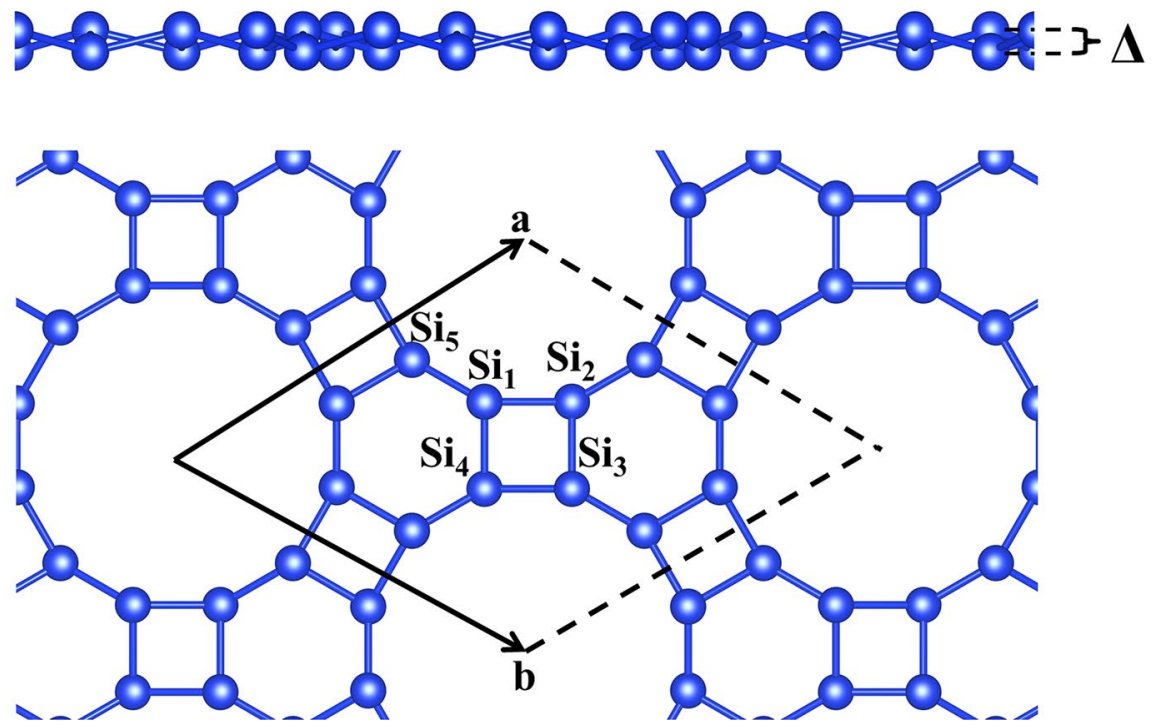
Table 1 Cell parameter ( $\mathrm{A})$, band gap energy (eV) and buckling $\Delta$ (A) of diamond silicon and silicene with their respective difference (\%) from the experimental/theoretical values (in parenthesis)

\begin{tabular}{llllll}
\hline & \multicolumn{2}{l}{ Silicon $(\Delta=0)$} & \multicolumn{2}{l}{ Silicene $\left(E_{\mathrm{g}}=0 \mathrm{eV}\right)$} \\
\cline { 2 - 3 } \cline { 5 - 6 } & $a$ & $E_{\mathrm{g}}$ & & \multicolumn{1}{l}{$\Delta$} \\
\hline BLYP & $5.523(1.7 \%)$ & $0.91(21.8 \%)$ & $3.890(1.6 \%)$ & $0.477(8.5 \%)$ \\
B3LYP & $5.475(0.8 \%)$ & $1.81(54.7 \%)$ & $3.863(0.9 \%)$ & $0.415(5.7 \%)$ \\
B3LYP* & $5.489(1.1 \%)$ & $1.43(22.2 \%)$ & $3.870(1.0 \%)$ & $0.439(0.2 \%)$ \\
B3LYP** & $5.499(1.3 \%)$ & $1.15(1.4 \%)$ & $3.876(1.2 \%)$ & $0.457(3.9 \%)$ \\
PBE & $5.459(0.5 \%)$ & $0.65(44.3 \%)$ & $3.860(0.8 \%)$ & $0.463(5.2 \%)$ \\
PBE0 & $5.423(0.1 \%)$ & $1.78(51.8 \%)$ & $3.842(0.3 \%)$ & 0.387 \\
PBESOL0 & $5.401(0.6 \%)$ & $1.68(43.5 \%)$ & $3.828(0.0 \%)$ & 0.387 \\
& & & & $(12.1 \%)$ \\
HSE06 & $5.427(0.1 \%)$ & $1.17(0.1 \%)$ & $3.840(0.3 \%)$ & $0.398(9.5 \%)$ \\
Exp. & $5.431[36]$ & $1.17[37]$ & - & - \\
Theo. & $5.382-5.467$ & - & $3.830[38]$ & $0.440[38]$ \\
& {$[42]$} & & & \\
\hline
\end{tabular}

per unit cell, and the SC belongs to the P622 space group with one irreducible atom per unit cell. All information about the unit cell of PS and SC structures is listed in Table S1 (Supplementary Material).

On the PS single-layer, the average length of intra-ring $\mathrm{Si}_{1}-\mathrm{Si}_{2}$ bond is $2.25 \AA$ and inter-ring $\mathrm{Si}_{3}-\mathrm{Si}_{4}$ bonds are $2.32 \AA$, and the buckling parameter is almost equal to the silicene, with a $0.01 \AA$ contraction. The $\mathrm{Si}-\mathrm{H}$ bond length is $1.49 \AA$, and the average bond angle centered on $\mathrm{Si}_{2}$ is $\sim 116^{\circ}$ and centered in $\mathrm{Si}_{3}$ is $\sim 120^{\circ}$. On the $\mathrm{SC}$, the cyclobutadienelike region is a quasi-perfect square with the $\mathrm{Si}_{2}-\mathrm{Si}_{3}$ and $\mathrm{Si}_{3}-\mathrm{Si}_{4}$ being 2.33 and $2.35 \AA$, respectively, with a bond angle of $\sim 87^{\circ}$; also the average intra-ring bond angles are $\sim 115^{\circ}$ and inter-ring bond angle are $\sim 140^{\circ}$; and in this structure the buckling is higher than the PS.

Upon the analysis of the overlap population, it can be observed that for PS, the overlap population on the inter-ring bonds $\left(\mathrm{Si}_{3}-\mathrm{Si}_{4}\right)$ is higher than the overlap on the intra-ring bonds $\left(\mathrm{Si}_{2}-\mathrm{Si}_{3}\right)$. For the $\mathrm{SC}$ structure, there are two different values of overlap for the inter-ring bonds, both higher than the intra-ring bonds. For both PS and SC structures, the inter-ring bond has a more covalent character than the intra-ring bonds.

As expected, the Mulliken charges of Si atoms in silicene and SC are uniformly distributed. In other way, on the PS structure there is a charge transfer from $\mathrm{Si}$ to $\mathrm{H}$ atom, and also a small charge dislocation from the $\mathrm{Si}$ adjacent to $\mathrm{Si}$ bonded with the $\mathrm{H}$.

Another important information to observe is that the PS has a smaller pore diameter (6.62 $\AA$ ) compared with SC, whose pore diameter is $8.63 \AA$, and it can be compared with the silicene pore which has a diameter of $4.5 \AA$. These $2 \AA$ of difference between the pores directly affect their applications, in particular, gas separation as a filtering membrane and battery anode as showed by $\mathrm{Hu}$ et al. [26, 27].

\subsection{Elastic properties}

Determining the mechanical properties may provide important information when external forces act on a material. The knowledge of the elastic properties can also be crucial to determinate the performance and the potential application of materials.

At first, the elastic constants for silicene (which represent the building block of nanoporous structures) were calculated in order to verify the numerical accuracy of the modified hybrid functional (B3LYP**) and of the adopted basis set.

The calculated elastic constants for silicene are $C_{11}=173.51 \mathrm{GPa}$ and $C_{12}=49.19 \mathrm{GPa}$. In order to compare the accuracy of the magnitude of the elastic properties with previous related results, the Young's modulus $(Y)$ and Poisson's ratio $(\nu)$ was calculated using the following expressions: $Y=\left(C_{11}^{2}-C_{12}^{2}\right) / C_{11}$ and $\nu=C_{12} / C_{11}$. Therefore, the Young's modulus and Poisson's ratio $(\nu)$ for silicene are $159.57 \mathrm{GPa}$ and 0.283 , respectively. These results are in good agreement with the reported theoretical values of the Young's modulus ( $148 \mathrm{GPa}$ ) [46] and Poisson's ratio (0.30) [46] of silicene.

The accuracy of the results for the mechanical properties for silicene should certify an accurate description for PS and SC structures, listed in Table 3.
Table 2 Cell parameters $(\AA)$, buckling $\Delta(\AA)$, bond length $(\AA)$, overlap population (in parenthesis) (mlel) and angles (deg) of PS and SC

\begin{tabular}{|c|c|c|c|c|c|c|}
\hline \multirow{2}{*}{$\begin{array}{l}a \\
\mathrm{PS}\end{array}$} & \multirow[t]{2}{*}{$b$} & \multirow[t]{2}{*}{$\Delta$} & \multicolumn{2}{|c|}{ Bond length (overlap) } & \multicolumn{2}{|l|}{ Angles } \\
\hline & & & & & & \\
\hline \multirow[t]{3}{*}{11.71} & \multirow[t]{3}{*}{11.71} & \multirow[t]{3}{*}{0.43} & $\mathrm{Si}_{1}-\mathrm{Si}_{2}$ & 2.25 (399) & $\mathrm{Si}_{1}-\mathrm{Si}_{2}-\mathrm{Si}_{3}$ & 117.41 \\
\hline & & & $\mathrm{Si}_{2}-\mathrm{H}$ & $1.49(321)$ & $\mathrm{H}-\mathrm{Si}_{2}-\mathrm{Si}_{3}$ & 115.02 \\
\hline & & & $\mathrm{Si}_{3}-\mathrm{Si}_{4}$ & $2.32(318)$ & $\mathrm{Si}_{2}-\mathrm{Si}_{3}-\mathrm{Si}_{4}$ & 119.88 \\
\hline \multicolumn{7}{|l|}{ SC } \\
\hline \multirow[t]{3}{*}{10.59} & \multirow[t]{3}{*}{10.59} & \multirow[t]{3}{*}{0.54} & $\mathrm{Si}_{1}-\mathrm{Si}_{2}$ & $2.25(354)$ & $\mathrm{Si}_{1}-\mathrm{Si}_{2}-\mathrm{Si}_{3}$ & 139.71 \\
\hline & & & $\mathrm{Si}_{2}-\mathrm{Si}_{3}$ & $2.35(267)$ & $\mathrm{Si}_{1}-\mathrm{Si}_{2}-\mathrm{Si}_{5}$ & 114.62 \\
\hline & & & $\mathrm{Si}_{3}-\mathrm{Si}_{4}$ & $2.33(302)$ & $\mathrm{Si}_{3}-\mathrm{Si}_{2}-\mathrm{Si}_{5}$ & 86.94 \\
\hline
\end{tabular}


Table 3 Elastic constants $C_{11}$ and $C_{12}(\mathrm{GPa})$, Young's modulus ( $Y$, $\mathrm{GPa})$ and Poisson's ratio $(\nu)$

\begin{tabular}{lrrrl}
\hline & \multicolumn{1}{c}{$C_{11}$} & \multicolumn{1}{c}{$C_{12}$} & \multicolumn{1}{l}{$Y$} & $v$ \\
\hline Silicene & 173.51 & 49.19 & 159.57 & 0.28 \\
PS & 72.97 & 23.36 & 65.49 & 0.32 \\
SC & 110.19 & 39.32 & 96.15 & 0.36 \\
Graphene [20] & 1025.92 & 163.01 & 1000.02 & 0.16 \\
PG [20] & 304.76 & 58.45 & 293.55 & 0.19 \\
GP [20] & 695.44 & 180.09 & 648.80 & 0.26 \\
\hline
\end{tabular}

The results show that silicene is more rigid than both nanoporous surfaces in both longitudinal and transversal direction. On the other hand, PS single-layer is more deformable than $\mathrm{SC}$, due to the strong bonding in the square ring of SC. Moreover, the Poisson's ratio for PS and SC, consequently, are higher than the silicene. Beyond that, in our previous work [20] it was presented a study of the elastic properties of graphene, porous graphene (PG) and graphenylene (GP), which are inserted in Table 3. Comparing these Young's modulus results with those obtained in the present work, it is possible to see that graphene is almost six times stiffer than silicene, the porous graphene is approximately $\sim 4.5$ times stiffer than porous silicene and graphenylene is approximately $\sim 6.7$ times stiffer than silicenylene. Furthermore, the Poisson's ratio of the silicene-based structures indicate that these structures are more deformable in the transversal direction, being more easy to break in a shear stress; and the PG and GP Poisson's ratio are similar to the pristine silicene.

\subsection{Electronic properties}

Figure 2 depicts the band structure, total and projected density of states of the silicene and both nanoporous surfaces.

The silicene (Fig. 2a) has zero band gap, with a welldefined Dirac cone, similar to the graphene, and located at $K$ point. PS (Fig. 2b) and SC (Fig. 2c) nanoporous surfaces have a direct band gap, $1.37 \mathrm{eV}$ and $76 \mathrm{meV}$, respectively, also located at $\mathrm{K}$ point.

It is evident that the pore leads a significant change in the electronic properties. Firstly, the band gap is opened from the silicene to PS. Second, occurs a sensible decrease in the band gap from PS to SC due to removal of the hydrogen atoms, leading to an structural rearrangements, which consist in an alternated rotation ( 30 degrees) of the hexagonal rings combined with the contraction of the lattice parameter $(\sim 10 \%)$ that forms a new cyclobutadiene-like ring between the rotated hexagonal rings and a increase in the buckling, which modifies the dihedral angle in $\sim 5^{\circ}$. New electronic states are created around the valence band (VB) and conduction band (CB), mainly associated with the $3 p_{\mathrm{z}}$ orbitals of the silicon atoms, as can observed in the density of states (Fig. 2).

Also is observed that the bands of PS are more flat than silicene; therefore, the electrons have a lower mobility in this region. On the other hand, SC shows only a small enlargement of the Dirac cone, creating non-flat bands, indicating a good dispersion and a higher mobility of the electrons; which could be interesting to electronic applications since this brings a small restrain to the electron mobility.

The DOS of PS (Fig. 2b) indicates that the main contribution to the VB and CB around the band gap comes from the $3 p_{\mathrm{z}}$ orbital of the silicon atoms, whereas there is no significant contribution from the $1 s$ orbital of the hydrogen atoms. According to Fig. 2c, the main contributor to both VB and $\mathrm{CB}$ of $\mathrm{SC}$ is also the $3 p_{\mathrm{z}}$ orbital of the silicon atoms.

\subsection{Raman spectra}

The Raman vibrational spectra and their most intense modes (without shifts) obtained for silicene, PS and SC are shown in Fig. 3.

These vibrational spectra can provide chemical and structural useful information to understand the experimental results, as well as a reference fingerprint for the structures.

The silicene shows a single Raman-active mode at $555.54 \mathrm{~cm}^{-1}$ (E, silicon asymmetric stretching), in agreement with the theoretical Raman G band at $\sim 550 \mathrm{~cm}^{-1}$. [47] SC has fifteen Raman-active modes, the most intense are located at $357.60 \mathrm{~cm}^{-1}\left(E_{2}\right.$, silicon symmetric stretching) and $394.14 \mathrm{~cm}^{-1}\left(E_{2}\right.$, silicon asymmetric stretching). The PS structure shows twenty-four Raman-active modes, the most intense of which are located at $504.13 \mathrm{~cm}^{-1}\left(A_{1}\right.$, silicon scissoring and hydrogen wagging), $549.00 \mathrm{~cm}^{-1}$ $\left(E_{2}\right.$, silicon asymmetric stretching and hydrogen rocking) and $723.01 \mathrm{~cm}^{-1}\left(E_{2}\right.$, carbon asymmetric stretching and hydrogen rocking). Also, for PS, there is only one active vibrational mode at frequencies above $2000 \mathrm{~cm}^{-1}$ which has a small intensity and only the hydrogen atoms oscillate with an asymmetric bond stretching. The theoretical vibrational modes are in agreement with experimental results for the $\mathrm{Si}-\mathrm{H}[48,49]$ and $\mathrm{Si}-\mathrm{Si}$ [50] bonding. The vibrational modes representation can be seen in the supplementary material.

\section{Conclusions}

Periodic DFT calculations were performed using BLYP, B3LYP, PBE, PBE0, PBESOL0, HSE06, modified B3LYP* (12\% hybrid) and modified B3LYP**(6\% hybrid) functional together with all-electron basis sets to simulate the structural, electronic, elastic and vibrational properties of diamond silicon, silicene, PS and SC single-layers 
Fig. 2 Band structure and total and projected density of states of a silicene, $\mathbf{b}$ PS and $\mathbf{c} \mathrm{SC}$
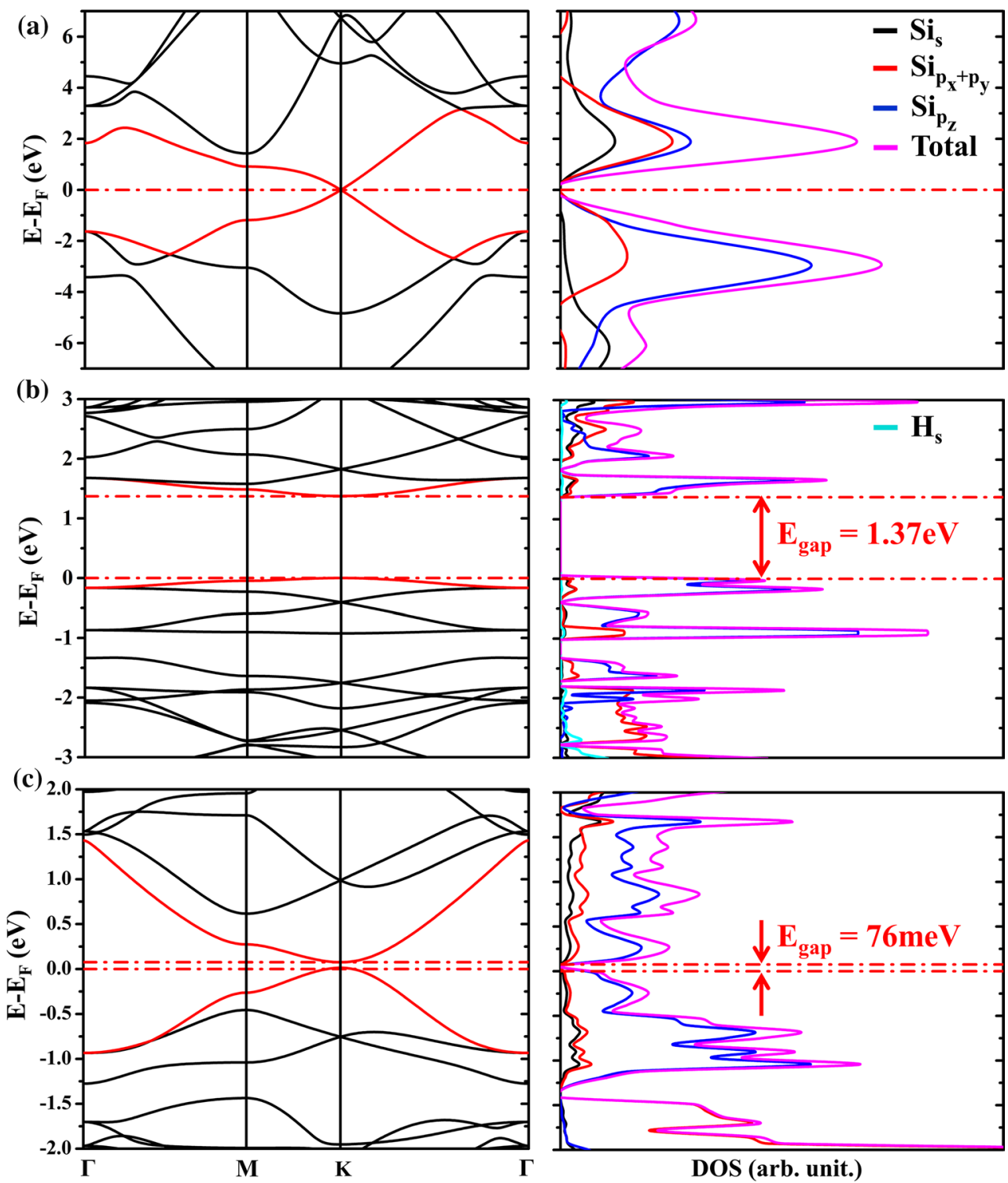

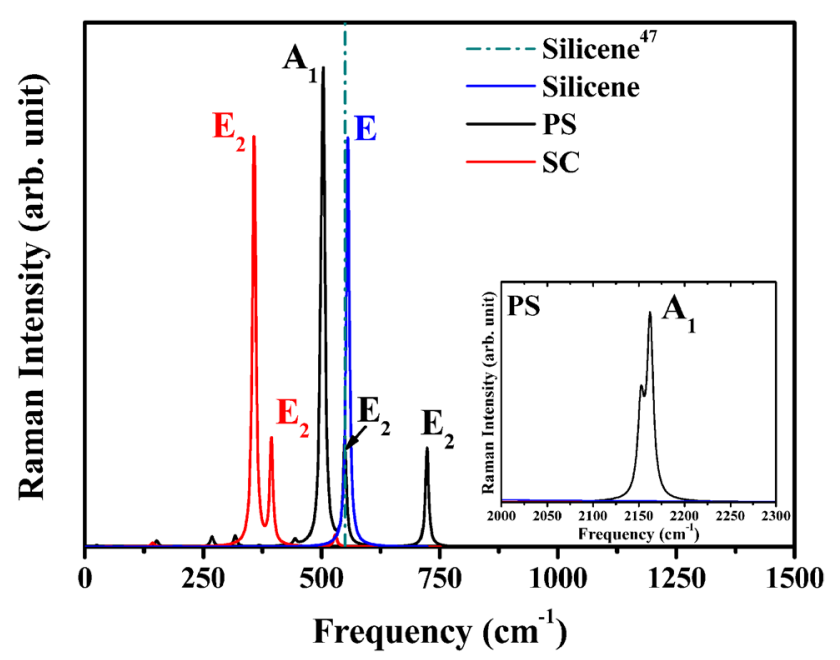

Fig. 3 Vibrational Raman spectra of silicene, PS, and SC structures surfaces. From all tested functional, the B3LYP** produced the most reliable results, with respect to the structural and electronic properties of diamond silicon and silicene single-layer surface; and thus it is expected that the prediction of the properties of PS and SC would have a good accuracy.

The analysis of the band structure shows that PS and SC have a direct band gap located at $K$ point. The pores significantly changed the electronic properties of the silicene. The band gap was opened from the silicene to PS, and the band gap dropped from the PS to SC, because of the dehydrogenation which leads to a structural rearrangements.

Besides, the SC shows non-flat band with an enlargement of the Dirac cone which gives greater mobility to the electrons with a small restrain to their movements, indicating a possible application on electronics, overcoming the problem with the high mobility of graphene and silicene. 
The analysis of the elastic properties indicates that PS and SC structures are more deformable in both longitudinal and transversal direction than silicene; consequently, the Poisson's ratio for both nanoporous structures is higher than silicene.

The Raman spectra of silicene are in good agreement with the other theoretical results, which allowed us to determine with reliability the Raman spectra of the PS and SC structures and determine their most intense vibrational modes.

The PS and SC structures can play an important role in improving the performance of nanosystems, applying this knowledge to electronics, gas filtering, adsorption systems and as an anode to lithium batteries. This could motivate further experimental and theoretical investigation in this field. Also it is expected that the generated geometric crystallographic information can be usefully applied to other studies.

Acknowledgements This work was supported by the Brazilian funding agencies CNPq (Grant No. 46126-4), CAPES (Grant Nos. 787027/2013, 8881.068492/2014-01), and FAPESP (Grant Nos. 2013/07296-2, 2016/07476-9, 2016/25500-4). The computational facilities were supported by resources supplied by the Molecular Simulations Laboratory, São Paulo State University, Bauru, Brazil. We would also like to thank Prof. Claudio Zicovich-Wilson (in memoriam) for the initial collaboration and suggestions.

\section{References}

1. Quhe R, Ma J, Zeng Z et al (2013) Tunable band gap in fewlayer graphene by surface adsorption. Sci Rep 3:1794. https://doi. org/10.1038/srep01794

2. Paupitz R, Autreto PAS, Legoas SB et al (2013) Graphene to fluorographene and fluorographane: a theoretical study. Nanotechnology 24:35706. https://doi.org/10.1088/0957-4484/24/3/035706

3. Stankovich S, Dikin DA, Piner RD et al (2007) Synthesis of graphene-based nanosheets via chemical reduction of exfoliated graphite oxide. Carbon 45:1558-1565. https://doi.org/10.1016 /j.carbon.2007.02.034

4. Flores MZS, Autreto PAS, Legoas SB, Galvao DS (2009) Graphene to graphane: a theoretical study. Nanotechnology 20:465704. https://doi.org/10.1088/0957-4484/20/46/465704

5. Ni ZH, Yu T, Lu YH et al (2008) Uniaxial strain on graphene: Raman spectroscopy study and band-gap opening. ACS Nano 2:2301-2305. https://doi.org/10.1021/nn800459e

6. Banhart F, Kotakoski J, Krasheninnikov AV (2011) Structural defects in graphene. ACS Nano 5:26-41. https://doi.org/10.1021 $/ \mathrm{nn} 102598 \mathrm{~m}$

7. Giovannetti G, Khomyakov PA, Brocks G et al (2008) Doping graphene with metal contacts. Phys Rev Lett 101:4-7. https://doi. org/10.1103/PhysRevLett.101.026803

8. Lalmi B, Oughaddou H, Enriquez H et al (2010) Epitaxial growth of a silicene sheet. Appl Phys Lett 97:223109. https://doi. org/10.1063/1.3524215

9. Aufray B, Kara A, Vizzini S et al (2010) Graphene-like silicon nanoribbons on $\operatorname{Ag}(110)$ : a possible formation of silicene. Appl Phys Lett 96:183102. https://doi.org/10.1063/1.3419932

10. Dávila ME, Xian L, Cahangirov S et al (2014) Germanene: a novel two-dimensional germanium allotrope akin to graphene and silicene. New J Phys 16:95002. https://doi.org/10.1088/1367-2630 /16/9/095002
11. Durgun E, Tongay S, Ciraci S (2005) Silicon and III-V compound nanotubes: structural and electronic properties. Phys Rev B 72:75420. https://doi.org/10.1103/PhysRevB.72.075420

12. Tao Y, Kanoh H, Abrams L, Kaneko K (2006) Mesopore-modified zeolites: preparation, characterization, and applications. Chem Rev 106:896-910. https://doi.org/10.1021/cr040204o

13. Stock N, Biswas S (2012) Synthesis of metal-organic frameworks (MOFs): routes to various MOF topologies, morphologies, and composites. Chem Rev 112:933-969. https://doi. org/10.1021/cr200304e

14. Sinha Ray S, Okamoto M (2003) Polymer/layered silicate nanocomposites: a review from preparation to processing. Prog Polym Sci 28:1539-1641. https://doi.org/10.1016/j.progpoly msci.2003.08.002

15. Lee J, Kim J, Hyeon T (2006) Recent progress in the synthesis of porous carbon materials. Adv Mater 18:2073-2094. https:// doi.org/10.1002/adma.200501576

16. Bieri M, Treier M, Cai J et al (2009) Porous graphenes: twodimensional polymer synthesis with atomic precision. Chem Commun. https://doi.org/10.1039/b915190g

17. Li Y, Zhou Z, Shen P, Chen Z (2010) Two-dimensional polyphenylene: experimentally available porous graphene as a hydrogen purification membrane. Chem Commun 46:3672. https://doi. org/10.1039/b926313f

18. Du A, Zhu Z, Smith SC (2010) Multifunctional porous graphene for nanoelectronics and hydrogen storage: new properties revealed by first principle calculations. J Am Chem Soc 132:2876-2877. https://doi.org/10.1021/ja100156d

19. Ding Y, Wang Y, Shi S, Tang W (2011) Electronic structures of porous graphene, $\mathrm{BN}$, and $\mathrm{BC} 2 \mathrm{~N}$ sheets with one- and twohydrogen passivations from first principles. J Phys Chem C 115:5334-5343. https://doi.org/10.1021/jp110336r

20. Fabris GSL, Marana NL, Longo E, Sambrano JR (2017) Theoretical study of porous surfaces derived from graphene and boron nitride. J Solid State Chem. https://doi.org/10.1016/j.jssc .2017.10.025

21. Brunetto G, Autreto PAS, Machado LD et al (2012) Nonzero gap two-dimensional carbon allotrope from porous graphene. J Phys Chem C 116:12810-12813. https://doi.org/10.1021/jp21 1300n

22. Totani R, Grazioli C, Zhang T et al (2017) Electronic structure investigation of biphenylene films. J Chem Phys 146:54705. http s://doi.org/10.1063/1.4975104

23. Du Q-S, Tang P-D, Huang H-L et al (2017) A new type of twodimensional carbon crystal prepared from 1,3,5-trihydroxybenzene. Sci Rep 7:40796. https://doi.org/10.1038/srep40796

24. Yu Y-X (2013) Graphenylene: a promising anode material for lithium-ion batteries with high mobility and storage. J Mater Chem A 1:13559. https://doi.org/10.1039/c3ta12639k

25. Song Q, Wang B, Deng K et al (2013) Graphenylene, a unique two-dimensional carbon network with nondelocalized cyclohexatriene units. J Mater Chem C 1:38-41. https://doi.org/10.1039/ C2TC00006G

26. Hu W, Wu X, Li Z, Yang J (2013) Helium separation via porous silicene based ultimate membrane. Nanoscale 5:9062. https://doi. org/10.1039/c3nr02326e

27. $\mathrm{Hu} \mathrm{W}, \mathrm{Wu} X, \mathrm{Li} \mathrm{Z}$, Yang J (2013) Porous silicene as a hydrogen purification membrane. Phys Chem Chem Phys 15:5753. https:// doi.org/10.1039/c3cp00066d

28. Zhuang J, Xu X, Peleckis G et al (2017) Silicene: a promising anode for lithium-ion batteries. Adv Mater. https://doi.org/10.1002 ladma.201606716

29. Zhang X, Qiu X, Kong D et al (2017) Silicene flowers: a dual stabilized silicon building block for high-performance lithium battery anodes. ACS Nano 11:7476-7484. https://doi.org/10.1021/ acsnano.7b03942 
30. Dovesi R, Orlando R, Erba A et al (2014) CRYSTAL14: a program for the ab initio investigation of crystalline solids. Int J Quantum Chem 114:1287-1317. https://doi.org/10.1002/qua.24658

31. Pascale $\mathrm{F}^{\dagger}$, Zicovich-Wilson $\mathrm{CM}^{\ddagger}$, Orlando $\mathrm{R}^{*}, \S$ et al (2005) Vibration Frequencies of Mg3A12Si3O12 Pyrope. An ab initio study with the CRYSTAL code. https://doi.org/10.1021/jp050316z

32. Dovesi R, Ermondi C, Ferrero E et al (1984) Hartree-Fock study of lithium hydride with the use of a polarizable basis set. Phys Rev B 29:3591-3600. https://doi.org/10.1103/PhysRevB.29.3591

33. Ferrero M, Rérat M, Kirtman B, Dovesi R (2008) Calculation of first and second static hyperpolarizabilities of one- to threedimensional periodic compounds. Implementation in the CRYSTAL code. J Chem Phys 129:244110. https://doi.org/10.1063 /1.3043366

34. Ferrero M, Rérat M, Orlando R, Dovesi R (2008) The calculation of static polarizabilities of 1-3D periodic compounds. the implementation in the crystal code. J Comput Chem 29:1450-1459. http s://doi.org/10.1002/jcc.20905

35. Ferrero M, Rérat M, Orlando R, Dovesi R (2008) Coupled perturbed Hartree-Fock for periodic systems: the role of symmetry and related computational aspects. J Chem Phys 128:14110. http s://doi.org/10.1063/1.2817596

36. Fujimoto H, Nakayama K, Tanaka M, Misawa G (1995) Absolute measurement of lattice spacing $\mathrm{d}(220)$ silicon crystal in floating zone. Jpn J Appl Phys 34:5065-5069. https://doi.org/10.1143/ JJAP.34.5065

37. Hybertsen MS, Louie SG (1985) First-principles theory of quasiparticles: calculation of band gaps in semiconductors and insulators. Phys Rev Lett 55:1418-1421. https://doi.org/10.1103/Phys RevLett.55.1418

38. Lew Yan Voon LC, Sandberg E, Aga RS, Farajian AA (2010) Hydrogen compounds of group-IV nanosheets. Appl Phys Lett 97:163114. https://doi.org/10.1063/1.3495786

39. Vogt P, De Padova P, Quaresima C et al (2012) Silicene: compelling experimental evidence for graphenelike two-dimensional silicon. Phys Rev Lett 108:155501. https://doi.org/10.1103/Phys RevLett.108.155501
40. Feng B, Ding Z, Meng S et al (2012) Evidence of silicene in honeycomb structures of silicon on $\mathrm{Ag}(111)$. Nano Lett 12:35073511. https://doi.org/10.1021/nl301047g

41. Fleurence A, Friedlein R, Ozaki T et al (2012) Experimental evidence for epitaxial silicene on diboride thin films. Phys Rev Lett 108:245501. https://doi.org/10.1103/PhysRevLett.108.245501

42. He L, Liu F, Hautier G et al (2014) Accuracy of generalized gradient approximation functionals for density-functional perturbation theory calculations. Phys Rev B 89:64305. https://doi.org/10.1103 /PhysRevB.89.064305

43 Kuzmin A, Kalinko A, Evarestov R (2011) First-principles LCAO study of phonons in NiWO4. Open Phys. https://doi.org/10.2478 /s11534-010-0091-z

44 Heyd J, Scuseria GE, Ernzerhof M (2003) Hybrid functionals based on a screened Coulomb potential. J Chem Phys 118:82078215. https://doi.org/10.1063/1.1564060

45 Krukau AV, Vydrov OA, Izmaylov AF, Scuseria GE (2006) Influence of the exchange screening parameter on the performance of screened hybrid functionals. J Chem Phys 125:224106. https:// doi.org/10.1063/1.2404663

46 Şahin H, Cahangirov S, Topsakal M et al (2009) Monolayer honeycomb structures of group-IV elements and III-V binary compounds: first-principles calculations. Phys Rev B 80:155453. http s://doi.org/10.1103/PhysRevB.80.155453

47 Kaloni TP, Cheng YC, Schwingenschlögl U (2013) Hole doped Dirac states in silicene by biaxial tensile strain. J Appl Phys 113:104305. https://doi.org/10.1063/1.4794812

48 Koshida N (2009) Device applications of silicon nanocrystals and nanostructures. Springer, Berlin. https://doi.org/10.1007/978-0387-78689-6

49 Fusina L, Cané E, Escribano R, Bürger H (1997) The Si-H stretching fundamentals of H328SiD. J Mol Spectrosc 184:385-394. http s://doi.org/10.1006/jmsp.1997.7340

50 Tolstoy VP, Chernyshova IV, Skryshevsky VA (2003) Handbook of infrared spectroscopy of Ultrathin films. https://doi.org/10.1002 /047123432x 